\title{
Étude Ethnobotanique et Screening Phytochimique de Quelques Ptéridophytes de la Forêt Classée de Yapo-Abbé (Côte d'Ivoire)
}

\author{
Adou Lydie Marie Dominique \\ Zaouri Bi Gala Jean-Marc
}

Laboratoire de Botanique, UFR Biosciences, Université Félix

HOUPHOUËT-BOIGNY, Abidjan, Côte d'Ivoire

\section{Bene Kouadio}

Laboratoire de Botanique et Phytothérapie, UFR des Sciences de la Nature,

Université Nangui Abrogoua, Abidjan, Côte d'Ivoire

\section{Zirihi Guédé Noël}

Laboratoire de Botanique, UFR Biosciences, Université Félix

HOUPHOUËT-BOIGNY, Abidjan, Côte d'Ivoire

Doi: 10.19044/esj.2018.v14n33p173 URL:http://dx.doi.org/10.19044/esj.2018.v14n33p173

\begin{abstract}
An ethnobotanical study with phytochemical screening of some Pteridophytes was carried out in the classified forest of Yapo-Abbe in the south of Côte d'Ivoire, in the Agboville Department, precisely in the subprefecture of Azaguié. The objective of this study was to show the different uses of the Pteridophytes of this forest. The approach method is an ethnobotanical survey carried out among the populations of the study area. This survey consisted in drafting a questionnaire beforehand to apprehend the multiple uses of Pteridophytes among the surrounding population and to develop a photo album of Pteridophytes to be presented during the interview. The data collection was carried out using the semi-structured interview method and with the help of an interpreter guide. It identified 19 species of Pteridophytes including six (6) species used as medicinal plants. No other use of these taxa was mentioned. The whole plant is the most solicited or $73.33 \%$ for the preparation of medicinal recipes. Various methods of preparation such as decoction (33.3\%), kneading (33.3\%), softening (20\%) and use in the fresh state $(13.3 \%)$ are used for recipes. single species. There are also different modes of administration of phytomedicaments: drink (42.85\%), local application (37.71\%), mastication (7.14\%) and massage (7.14\%). The phytochemical sorting performed on the hydroethanolic extracts revealed that the different drugs of these plants contain sterols, polyterpenes, polyphenols,
\end{abstract}


flavonoids, catechin tannins, alkaloids and quinones which give them various therapeutic properties.

Keywords: Côte d'Ivoire, Medicinal plant, Pteridophytes, Nephrolepis biserrata, Phytochemistry, Chemical compound

\section{Résumé}

Une étude ethnobotanique avec screening phytochimique de quelques Ptéridophytes a été réalisée dans la forêt classée de Yapo-Abbé au Sud de la Côte d'Ivoire, dans le Département d'Agboville, précisément dans la souspréfecture d'Azaguié. L'objectif de cette étude était de montrer les différents usages des Ptéridophytes de cette forêt. La méthode d'approche est une enquête ethnobotanique réalisée auprès des populations de la zone d'étude. Cette enquête a consisté à rédiger au préalable un questionnaire permettant d'appréhender les multiples usages des Ptéridophytes auprès de la population environnante et à élaborer un album photos de Ptéridophytes à présenter lors de l'entretien. La collecte de données a été effectuée par la méthode des entretiens semi-structurés et avec l'aide d'un guide interprète. Elle a permis de recenser 19 espèces de Ptéridophytes dont six (6) espèces utilisées comme plantes médicinales. Aucun autre usage de ces taxons n'a été mentionné. La plante entière est la plus sollicitée soit 73,33 \% pour la préparation des recettes médicamenteuses. Divers modes de préparation que sont la décoction $(33,3 \%)$, le pétrissage $(33,3 \%)$, le ramollissement $(20 \%)$ et l'utilisation à l'état frais $(13,3 \%)$ sont utilisés pour des recettes monospécifiques. On note également différents modes d'administration des phytomédicaments : boisson $(42,85 \%)$, application locale $(37,71 \%)$, mastication $(7,14 \%)$ et massage $(7,14 \%)$. Le tri phytochimique réalisé sur les extraits hydroéthanoliques a révélé que les différentes drogues de ces plantes renferment des stérols, des polyterpènes, des polyphénols, des flavonoïdes, des tanins catéchiques, des alcaloïdes et des quinones qui leur confèrent les propriétés thérapeutiques diverses.

Mots clés: Côte d'Ivoire, Plantes médicinales, Pteridophytes, Nephrolepis biserrata Phytochimie, Composés chimiques

\section{Introduction}

La nature est, et demeure une source intarissable où l'Homme puise nourriture et remèdes pour son bien-être (Enda Tier-monde, 1986). Les plantes ont été depuis les temps anciens un élément de choix de lutte pour l'homme contre les agressions de toutes sortes. Ces plantes cultivées ou non fournissent à l'homme la plus grande part de son alimentation, de son artisanat, de ses vêtements, de son habitation, de son horticulture, de sa médecine, de sa chasse, 
de sa pêche, de sa cosmétique, de son énergie, de sa zootechnie, en un mot les matières premières de ses diverses industries traditionnelles (N'Guessan, 1995). Ces usages varient en fonction des plantes que l'on a à sa disposition (Mangambu et al., 2012).

L'étude des usages et des relations entre les plantes et les humains constitue l'ethnobotanique. Pendant plusieurs décennies, les études ethnobotaniques ont été basées essentiellement sur les Spermaphytes (Singh et al., 2003). Mais actuellement, ces études ont été élargies à d'autres embranchements du règne végétal. Ainsi, depuis environ une décennie, les études ethnoptéridologiques ont plus souvent été abordées un peu partout dans le monde (Asie mineure (Lognay et al., 2008 ), Inde (Singh et al, 2012 ; Dhiman, 1998 ), RD Congo (Mangambu et al., 2012) et incitent plusieurs chercheurs à comprendre l'usage des plantes de cet embranchement par différents groupes ethno-linguistiques et les interactions entre l'environnement naturel et les peuples autochtones (Rout et al., 2009)

Les Ptéridophytes ou Cryptogames vasculaires constituent un groupe primitif intermédiaire entre les végétaux non vasculaire (Thallophytes, Bryophytes) et les végétaux supérieurs pleinement adaptés à la vie terrestre que sont les Spermaphytes (Adou, 2007).

En Afrique, précisément au Congo, ces taxons sont bien connus et utilisés dans plusieurs domaines (Mangambu et al., 2012). En Côte d'Ivoire, ces végétaux ont été étudiés par divers chercheurs dont Aké-assi, (1984), TraBi (1993), Adou (2000, 2007). Ces travaux réalisés sont presque essentiellement des inventaires. Plus rarement, il a été question d'ethnobotanique Adou et al., (2014, 2016 d); (Goneziety, 2016). Cependant, il ne faut pas oublier de citer les travaux de Kerharo et Bouquet (1950) et ceux de Bouquet et Debray (1974) qui ont fait des études sur les plantes médicinales de Côte d'Ivoire en incluant les Ptéridophytes. Dans ce pays, les Ptéridophytes sont jusqu'à présents peu connus, car peu sinon pas du tout étudiés.

Il devient donc impérieux de chercher à les valoriser afin de pouvoir les protéger. Ce travail s'inscrit dans une connaissance générale des Ptéridophytes de Côte d'Ivoire. Il a pour objectif de montrer les différents usages des Ptéridophytes de la forêt classée de Yapo-Abbé (FCYA) et de déterminer les différents groupes chimiques contenus dans les plantes signalées comme médicinales.

\section{Matériel et Méthodes \\ Cadre d'étude}

Située dans la région Sud de la Cote d'Ivoire (Figure 1) dans le domaine guinéen, la forêt classée de Yapo-Abbé d'une superficie de 29795,37 ha est localisée dans la Sous-préfecture d'Azaguié, dans le Département d'Agboville. Comprise entre $5^{\circ} 40^{\prime} 02$ et $5^{\circ} 47^{\prime} 32$ de latitudes Nord, $3^{\circ} 57^{\prime}$ 
02 et $4^{\circ} 11^{\prime} 37$ de longitudes Ouest, cette forêt se trouve à $8 \mathrm{~km}$ au Nord d'Azaguié, à $25 \mathrm{~km}$ au sud d'Agboville, et à $50 \mathrm{~km}$ au Nord-Est d'Abidjan, sur la route d'Adzopé. Cette localité est peuplée en majorité par les Abbey, les Malinké, les Baoulé, les Attié et d'autres ressortissants de la sous-région (Bodoua, 2013).

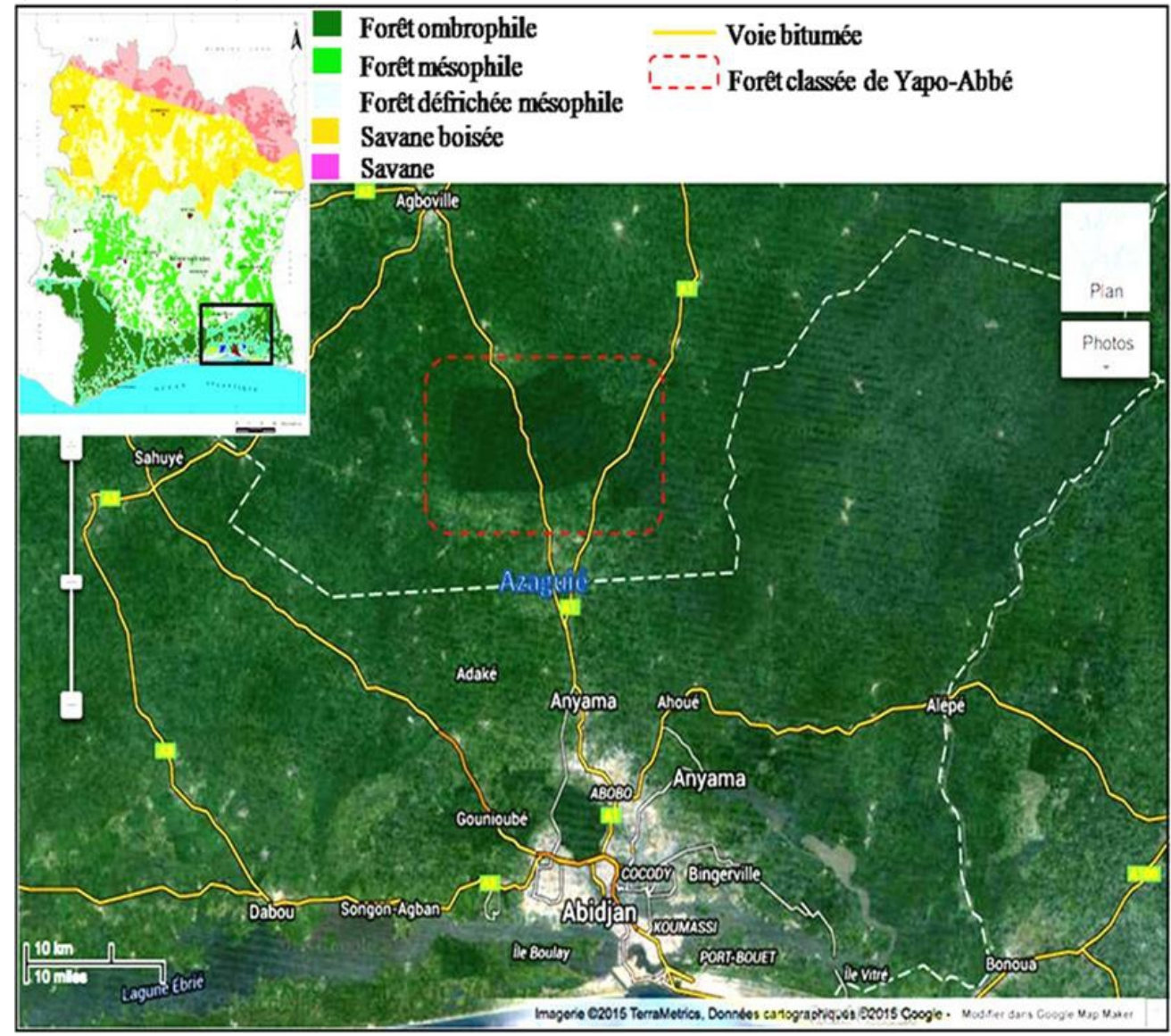

Figure 1 : Localisation géographique de la forêt classée de Yapo-Abbé

(Source : Piba et al., 2015)

\section{Matériel}

\section{Matériel biologique (végétal)}

Le matériel végétal est constitué de l'ensemble des Ptéridophytes récoltées. Aussi diverses drogues de certaines espèces ont été pulvérisées et utilisées pour la préparation des extraits (éthérique, méthanolique et aqueux) concernant l'étude tri phytochimique.

\section{Matériel technique}

Pour l'enquête Ethnobotanique, le matériel classique a été utilisé. Il s'agit entre autres de fiches d'enquête, d'un carnet de note, d'un appareil 
photographique numérique. Pour la récolte des échantillons, des machettes et un sécateur ont été utilisés. Concernant le screening phytochimique, nous avons utilisés des tubes à essai, des micropipettes et des microplaques, des éprouvettes graduées, d'entonnoirs, des papiers filtres, du coton hydrophile, un broyeur électrique, du papier aluminium, une étuve, des assiettes en porcelaine, un bain marie, et un bain de sable. Ce matériel comportait également des solvants et réactifs pour mettre en évidence la présence des grands groupes phytochimiques.

Pour la caractérisation des groupes chimiques, le matériel utilisé par Doga, 2017 a été sollicité. Ainsi pour la recherche des stérols et polyterpènes, les réactifs utilisés sont l'anhydride acétique et l'acide sulfurique concentré. Le Chlorure ferrique $\left(\mathrm{Fecl}_{3}\right)$ à $2 \%$ a permis la caractérisation des polyphénols . L'alcool chlorhydrique dilué 2 fois, les copeaux de magnésium et 1'alcool isoamylique ont été utilisés pour rechercher les flavonoïdes. La caractérisation des tanins catéchiques a été mise en évidence par le Formaldéhyde et l'Acide chlorhydrique concentré. Quant aux tanins galliques, nous avons employé l'acétate de sodium et le chlorure ferrique. L'Ammoniaque $(0,1 \mathrm{~N})$ a permis de rechercher les quinones. Enfin, la caractérisation des alcaloïdes a été faite par le réactif de Dragendorff (solution iodo-bismutathe de potassium) et le réactif de Burchard.

\section{Méthodes}

La méthode utilisée est inspirée de celle de Maregesi et al. (2007). Elle a consisté en premier lieu à rédiger un questionnaire permettant d'appréhender les multiples usages des Ptéridophytes auprès de la population environnante (Malay, 2011 ; Singh et al., 2003). Ces questionnaires visaient à interroger les habitants de la localité, les personnes âgées et les détenteurs de connaissances ancestrales. Les interviews ont été faites avec un guide interprète au cas où on rencontrerait des personnes ne comprenant pas français.

La collecte de données a été effectuée par la méthode des entretiens semi-structurés (Kakudidi, 2004). Des échantillons de Ptéridophytes accompagnés de photos ont été présentés lors des entretiens. Pour chaque plante recensée, le nom vernaculaire, l'organe utilisé ainsi que son usage ont été demandés. Les localités choisies pour les enquêtes sont Azaguié, YakasséMé et Agboville. Les enquêtes ce sont déroulées de juillet à Août. Cette période correspond à la petite saison sèche. Quant aux personnes interviewées (Hommes et femmes de 25 à 70 ans) au nombre de 30, étaient en grande majorité des Abbey et des Attiés. Ils sont soit tradi-thérapeutes, herboristes ou détenteurs de connaissances ancestrales. Les échantillons récoltés ont été identifiés au Centre National de Floristique (CNF).

Des drogues végétales ont été récoltées puis séchées au laboratoire à l'abri du soleil à la température ambiante. Après deux (2) semaines de séchage 
elles ont été pulvérisées à l'aide d'un broyeur électrique. Chaque fine poudre (broyat) a subi une extraction selon la deuxième variante de la méthode de Zirihi et al. (2003). Ainsi, $100 \mathrm{~g}$ de broyat ont été macérés dans 1L d'alcool éthylique à $96^{\circ}$ dilué à $70 \%$ pendant 5 min dans un Blinder. Le mélange est ensuite filtré trois fois, d'abord à l'aide d'un carré de tissu ensuite sur du coton hydrophyle pour un second filtrage et finalement à l'aide un filtre à café pour le troisième et dernier filtrage. L'opération est répétée comme précédemment mais cette fois en utilisant le mac. Le filtrat recueilli, a été évaporé à l'étuve à $50{ }^{\circ} \mathrm{C}$. La poudre ainsi obtenue est l'extrait hydro-alcoolique.

Un tri phytochimique a été par la suite réalisé afin de mettre en évidence la présence de grands groupes de métabolites secondaires des plantes sélectionnées. La caractérisation des groupes chimiques résumée dans le tableau I a été faite selon la méthode utilisée par Doga (2017).

Tableau I : Tableau synthétiques des groupes chimiques, réactions d'identification et indicateurs (Source : Doga, 2017)

\begin{tabular}{|c|c|c|c|}
\hline \multicolumn{2}{|c|}{ Groupes chimiques } & Réactifs d'identifications & Indicateurs (réaction positive) \\
\hline \multicolumn{2}{|c|}{ Stérols et polyterpènes } & $\begin{array}{c}\text { Anhydride acétique } \\
\text { Acide sulfurique concentré }\end{array}$ & $\begin{array}{c}\text { Apparition à l'interphase d'un anneau } \\
\text { pourpre ou violet, virant au bleu puis } \\
\text { au vert }\end{array}$ \\
\hline \multicolumn{2}{|c|}{ Polyphénols } & Chlorure ferrique $\left(\mathrm{Fecl}_{3}\right)(2 \%)$ & $\begin{array}{l}\text { Apparition d'une coloration bleu- } \\
\text { noirâtre ou verte plus ou moins foncée }\end{array}$ \\
\hline \multicolumn{2}{|c|}{ Flavonoïdes } & $\begin{array}{c}\text { Alcool chlorhydrique } \\
\text { Copaux de magnésium } \\
\text { Alcool isoamylique } \\
\end{array}$ & $\begin{array}{l}\text { Dégagement de chaleur puis coloration } \\
\text { rose-orangé ou violacée }\end{array}$ \\
\hline \multirow{2}{*}{ Tanins } & Catéchiques & $\begin{array}{c}\text { Formaldéhyde } \\
\text { Acide chlorhydrique concentré }\end{array}$ & Précipité gélatineux (en gros flocons) \\
\hline & Galliques & $\begin{array}{c}\text { Acétate de sodium } \\
\text { Chlorure ferrique }\end{array}$ & Coloration bleu-noir intense \\
\hline \multicolumn{2}{|c|}{ Quinones } & Ammoniaque $(0,1 \mathrm{~N})$ & $\begin{array}{l}\text { Apparition d'une coloration allant du } \\
\text { rouge au violet }\end{array}$ \\
\hline \multirow{2}{*}{\multicolumn{2}{|c|}{ Alcaloïdes }} & $\begin{array}{l}\text { Dragendorff (solution iodo-bismutathe de } \\
\text { potassium) }\end{array}$ & \multirow{2}{*}{$\begin{array}{l}\text { Précipitation de coloration brun- } \\
\text { rougeâtre }\end{array}$} \\
\hline & & Burchard (Réaction iodo- isodurée) & \\
\hline
\end{tabular}

\section{Résultats et Discussion Enquête ethnobotanique}

Les investigations botaniques menées dans la forêt classée Yapo-Abbé ont permis d'inventorier dix-neuf (19) espèces de Ptéridophytes réparties entre quinze (15) genres et douze (12) familles botaniques (Tableau II). 
Tableau II : Ptéridophytes de la forêt classée Yapo-Abbé

\begin{tabular}{|c|l|l|}
\hline $\mathbf{N}^{\circ}$ & \multicolumn{1}{|c|}{ Nom de l'espèce } & \multicolumn{1}{|c|}{ Familles } \\
\hline 1 & Adiantum vogelii Mett. ex Keys & Adiantaceae \\
\hline 2 & Asplenium africanum Desv. & Aspleniaceae \\
\hline 3 & Ctenitis protensa (Afz. ex Sw.) Ching & Dryopteridaceae \\
\hline 4 & Ctenitis pilosissima (J.Sm.) Alston & Dryopteridaceae \\
\hline 5 & Cyclosorus dentatus ( Forsk.) Ching & Thelypteridaceae \\
\hline 6 & Cyclosorus striatus (Schum.) Ching & Thelypteridiaceae \\
\hline 7 & Gleichenia linearis (Burm.)C.B. Clarke & Gleicheniaceae \\
\hline 8 & Huperzia staudii (Nessel) Pic. Serm. & Lycopodiaceae \\
\hline 9 & Lygodium microphyllum (Cav.)R.Br. & Lygodiaceae \\
\hline 10 & Lygodium smithianum Presl. ex Kühn & Lygodiaceae \\
\hline 11 & Microgramma owariensis (Desv.) Alston & Polypodiaceae \\
\hline 12 & Microsorum punctatum (L.) Copel & Polypodiaceae \\
\hline 13 & Nephrolepis biserrata (Sw.) Schott & Nephrolepidaceae \\
\hline 14 & Phymatodes scolopendria (Burm.) Ching & Polypodiaceae \\
\hline 15 & Pityrogramma calomelanos (L.) Link & Pteridaceae \\
\hline 16 & Platycerium stemaria (P.Beauv.) Desv & Polypodiaceae \\
\hline 17 & Pteris atrovirens Willd. & Pteridaceae \\
\hline 18 & Pteris burtoni Bak. & Pteridaceae \\
\hline 19 & Selaginella myosorus (Sw.)Alston & Selaginellaceae \\
\hline & & \\
\hline
\end{tabular}

Parmi celles-ci, six (6) espèces sont utilisées comme plantes médicinales dans la Forêt Classée de Yapo-Abbé. Ces six espèces sont: Cyclosorus striatus (Schumach) Ching, Microgramma owariensis (Desv.) Alston, Microsorium punctatum (L.) Copel, Nephrolepis biserrata (Sw.) Schott, Phymatodes scolopendria (Burm.) Ching et Selaginella myosorus (Sw.) Alston. La fréquence de citation de chaque espèce a été établie (Figure 2).

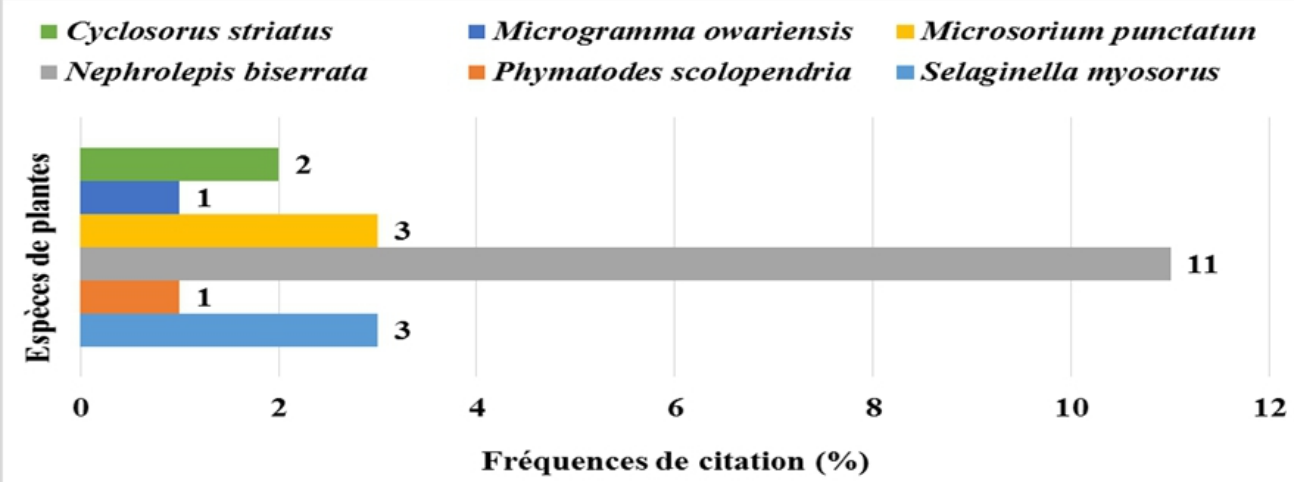

Figure 2 : Fréquence de citation des six espèces médicinales 
Les six espèces de Ptéridophytes utilisées comme plantes médicinales dans cette région sont réprésentées sur cette figure en fonction des fréquences de citation. Nephrolepis biserrata citée 11 fois soit 52,38 \% est l'espèce la plus citée par les personnes interviewées. Cependant, nous n'avons pas reçues d'informations nous disant que cette espèce était la plus utilisée. Phymatodes scolopendria et Microgramma owariensis citées chacune 1 fois soit 4,76\% sont les moins citées.

Il ressort de l'étude que, seul l'usage médicinal des Ptéridophytes a été signalé par les populations environnantes de la forêt classée de Yapo-Abbé (FCYA). Ces résultats ne concordent pas avec ceux de Gonezieti (2016) qui a travaillé sur les utilisations des Ptéridophytes du Parc National du Banco (Côte d'Ivoire). Avec cet auteur, plusieurs domaines d'utilisations de ces taxons ont été signalés. Cela pourrait s'expliquer par le fait que la forêt du banco étant une zone urbaine, les besoins en produits forestiers sont forts et diversifiés, alors que la présente étude a été faite en zone rurale. Cet usage unique pourrait également s'expliquer par le fait que la FCYA regorge une diversité de ressources ligneuses qui pourvoient aux besoins de la population. Du coup, les produits non ligneux qui sont d'abord rares, difficiles à récolter, surtout les épiphytes, ne sont plus recherchées. En effet, les travaux de Piba et al. (2015) sur la disponibilité des espèces sources de produits forestiers non ligneux (PFNL) ont montré que dans l'ensemble, la FCYA renferme des plantes médicinales. Cependant, les investigations dans ce domaine ont porté essentiellement sur les végétaux supérieurs. La non utilisation des Ptéridophytes recensées comme plante alimentaire chez l'Homme se justifierait par le fait qu'elles ne font pas partie du régime alimentaire des habitants de cette localité ou encore par le fait que certaines pourraient être cancérigènes (Lukavsky, 1996). Le fait qu'elles ne soient pas également signalées comme plantes fourragères pourrait être dû à la nocivité potentielle des fougères et aussi à cause du fait que la population de la localité n'a pas d'activité pastorale.

Dans le secteur d'étude, les Ptéridophytes sont sollicitées pour plusieurs thérapies. Le tableau III présente les indications thérapeutiques, les parties utilisées, les modes de préparation et d'administration dans l'usage médicinales des Ptéridophytes. 
Tableau III : Ptéridophytes et indications thérapeutiques

\begin{tabular}{|c|c|c|c|c|}
\hline $\begin{array}{c}\text { Noms } \\
\text { scientifiques }\end{array}$ & $\begin{array}{l}\text { Indications } \\
\text { thérapeutiques }\end{array}$ & $\begin{array}{l}\text { Parties } \\
\text { utilisées }\end{array}$ & $\begin{array}{l}\text { Modes de } \\
\text { préparation du } \\
\text { remède }\end{array}$ & Modes d'administration \\
\hline \multirow{2}{*}{$\begin{array}{l}\text { Cyclosorus } \\
\text { striatus } \\
\text { (Schumach) } \\
\text { Ching } \\
\end{array}$} & L'asthme & Plante entière & Pétrissage & \multirow[b]{2}{*}{ Boisson } \\
\hline & $\begin{array}{l}\text { L'hypertension } \\
\text { Artérielle }\end{array}$ & Plante entière & Décoction & \\
\hline \multirow{7}{*}{$\begin{array}{l}\text { Nepphrolepis } \\
\text { biserrata (Sw.) } \\
\text { Schott }\end{array}$} & $\begin{array}{l}\text { Douleurs de dos } \\
\text { et de nerfs }\end{array}$ & Plante entière & Ramollissement & Massage \\
\hline & L'Asthme & Feuille & Pétrissage & Boisson \\
\hline & $\begin{array}{l}\text { L'hypertension } \\
\text { Artérielle }\end{array}$ & Plante entière & Décoction & Boisson \\
\hline & Ulcère de burili & Feuille & Ramollissement & $\begin{array}{c}\text { Application sur la partie } \\
\text { malade }\end{array}$ \\
\hline & Aphrodisiaque & Plante entière & Frais & Mastication \\
\hline & Empoisonnement & Plante entière & Décoction & Boisson \\
\hline & Enflures & Feuille & Ramollissement & $\begin{array}{l}\text { Application sur la partie } \\
\text { malade }\end{array}$ \\
\hline \multirow{3}{*}{$\begin{array}{l}\text { Microsorium } \\
\text { punctatum (L.) } \\
\text { Copel }\end{array}$} & Paralysie & Plante entière & Pétrissage & $\begin{array}{c}\text { Application sur la partie } \\
\text { paralysée }\end{array}$ \\
\hline & $\begin{array}{l}\text { Insuffisance de } \\
\text { lait dans les seins } \\
\text { de la mère après } \\
\text { accouchement }\end{array}$ & Plante entière & Pétrissage & Application sur les seins \\
\hline & Rhumatisme & Plante entière & Pétrissage & Application sur tout le corps \\
\hline $\begin{array}{l}\text { Microgramma } \\
\text { owariensis } \\
\text { (Desv.) Alston }\end{array}$ & Paludisme & Feuille & Décoction & Boisson \\
\hline $\begin{array}{l}\text { Phymatodes } \\
\text { scolopendria } \\
\text { (Burm.) Ching }\end{array}$ & Paludisme & Plante entière & Décoction & Boisson \\
\hline $\begin{array}{l}\text { Selaginella } \\
\text { myosorus } \\
\text { (Sw.) Alston }\end{array}$ & $\begin{array}{c}\text { Contre les } \\
\text { mauvais esprits } \\
\text { sur les jumeaux }\end{array}$ & Plante entière & Frais & $\begin{array}{c}\text { Dépôt aux cheveux des } \\
\text { jumeaux }\end{array}$ \\
\hline
\end{tabular}

L'analyse de ce tableau révèle que la plante entière et les feuilles sont les seules parties sollicitées pour la préparation des recettes médicamenteuses avec une prédominance pour l'usage de la plante entière (73\%) (Figure 3). Ces résultats ne concordent pas avec ceux des travaux effectués par N'Guessan et al. (2009) et Offoumou (2015) qui ont montré que les feuilles constituaient la partie la plus sollicitée avec respectivement $51,2 \%$ et $49,3 \%$. Ce qui pourrait s'expliquer par le fait que ces derniers ont travaillé sur des Phanérogames qui sont des plantes de grandes tailles alors que ce travail porte sur les Ptéridophytes qui sont en général de petites tailles. Cependant, ces résultats 
sont beaucoup plus proches de ceux de Gonezieti (2016) (61,11\%) qui a également travaillé sur les Ptéridophytes dans le Parc National du Banco.

Figure 3 : Spectre des différentes parties des plantes utilisées pour la préparation des recettes

La figure 4 présente les histogrammes de fréquence des différents modes de préparation des remèdes.

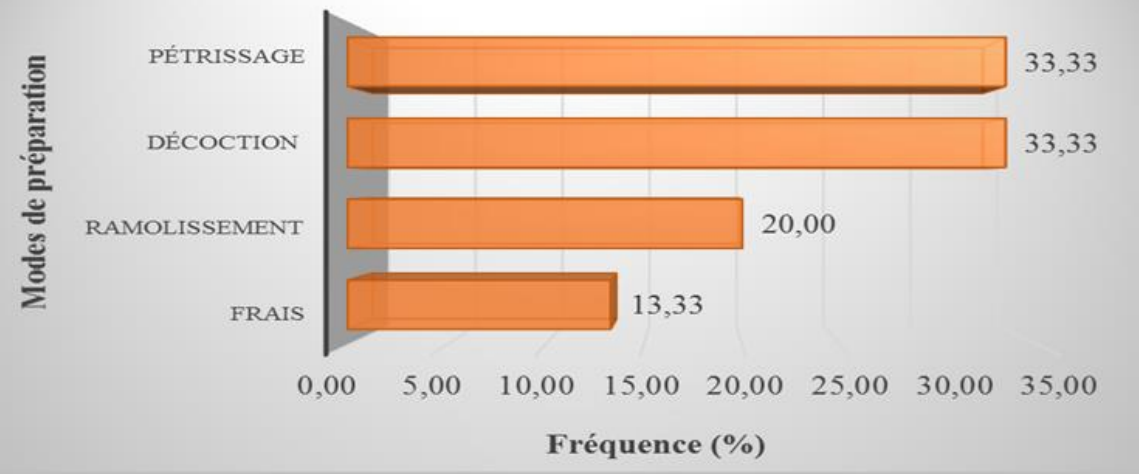

Figure 4 : Histogrammes de fréquence des différents modes de préparation des remèdes

Plusieurs modes de préparation médicamenteuses sont employées. La décoction $(33,3 \%)$ et le pétrissage $(33,3 \%)$ sont les plus sollicités. Viennent ensuite le ramollissement $(20 \%)$ et enfin l'utilisation à l'état frais $(13,33 \%)$. Le mode de préparation des médicaments est rattaché aux mœurs de chaque peuple, il dépend également du type d'affection traitée (Doga, 2017). Ces résultats concordent plus ou moins avec ceux de Goneziéti (2016) qui a montré que la décoction $(37,1 \%)$ et le pétrissage $(29,6 \%)$ étaient les plus sollicités. Cependant ces résultats sont différents de ceux de (N'Guessan et al., 2009) qui soutiennent que le pétrissage est le plus employé chez les Abbey et les Krobou d'Agboville. Selon Salhi et al. (2010), la décoction permet de recueillir le plus de principes actifs et atténue ou annule l'effet toxique de certaines recettes.

La figure 5 ci- dessous présente les modes d'administration des phytomédicaments. 


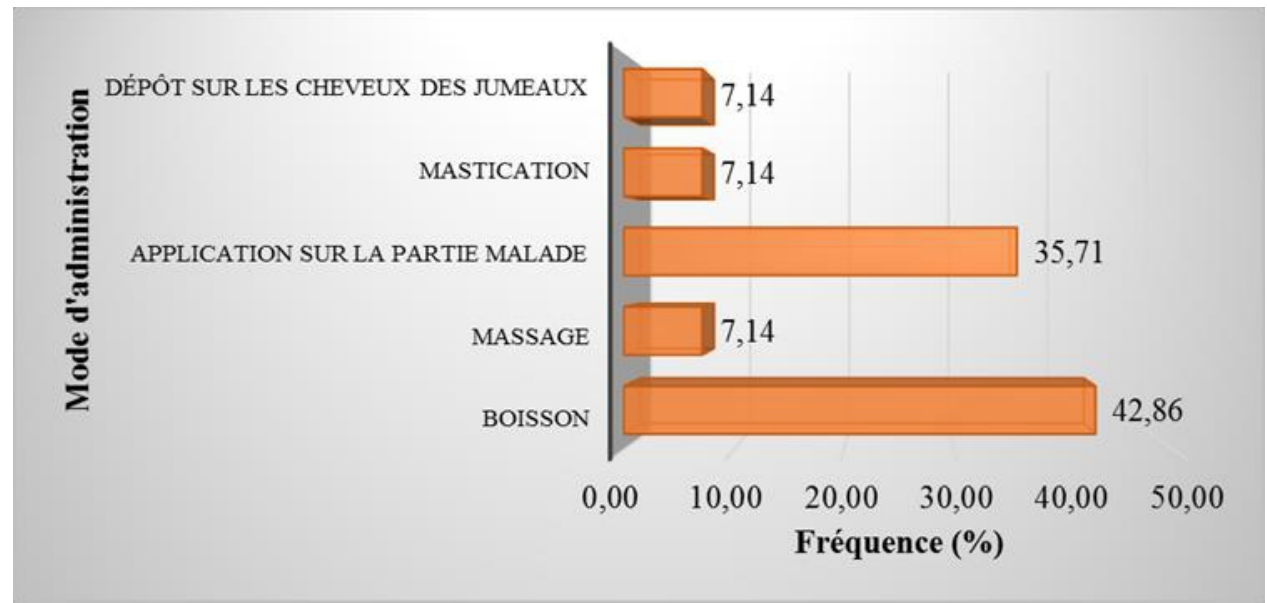

Figure 5 : Histogrammes de fréquence des modes d'administration

Divers modes d'administration (boisson, massage, application sur la partie malade, etc.), sont utilisés. La boisson $(42,85 \%)$ est le mode le plus employé. Ensuite vient le mode application sur la partie malade $(37,71 \%)$. Cela s'expliquerait par le fait que ces pratiques demeurent les plus faciles. Ces résultats ne concordent pas avec ceux de Goneziéti (2016) dont le travail avait porté sur le même groupe de végétaux.

\section{Mise en évidence des groupes chimiques responsables des effets thérapeutiques}

Le tri phytochimique a permis de mettre en évidence la présence des principaux groupes chimiques dans les extraits hydroalcooliques. L'exploration phytochimique a porté sur six Ptéridophytes sélectionnées. Les résultats sont consignés dans le tableau IV.

Tableau IV : Tri phytochimique des espèces sélectionnées

\begin{tabular}{|l|c|c|c|c|c|c|c|c|}
\hline \multirow{2}{*}{$\begin{array}{c}\text { Plantes } \\
\text { PPtéridophytes } \\
\text { sélectionnées) }\end{array}$} & \multirow{2}{*}{$\begin{array}{c}\text { Stérols et } \\
\text { Polyterpènes }\end{array}$} & \multirow{2}{*}{ Polyphénols } & Flavonoïdes & \multicolumn{2}{|c|}{ Tanins } & \multicolumn{2}{|c|}{ Alcaloüdes } \\
\cline { 6 - 9 } & & & Cat & Gal & & Quinones & D \\
\hline $\begin{array}{l}\text { Cyclosorus dentatus } \\
\text { (Forssk.) R. C. Ching }\end{array}$ & ++ & - & - & - & - & - & ++ & ++ \\
\hline $\begin{array}{l}\text { Microgramma } \\
\text { owariensis (Desv.) } \\
\text { Alston }\end{array}$ & ++ & + & + & + & - & - & ++ & ++ \\
\hline $\begin{array}{l}\text { Microsorium } \\
\text { punctatum (L.) Copel }\end{array}$ & ++ & ++ & ++ & + & - & - & ++ & ++ \\
\hline $\begin{array}{l}\text { Nephrolepis biserrata } \\
\text { (Sw.) Schott }\end{array}$ & ++ & + & + & + & - & - & ++ & ++ \\
\hline $\begin{array}{l}\text { Phymatodes } \\
\text { scolopendria (Burm.) } \\
\text { Ching }\end{array}$ & ++ & ++ & ++ & + & - & - & ++ & ++ \\
\hline $\begin{array}{l}\text { Selaginella myosorus } \\
\text { (Sw.) Alston }\end{array}$ & ++ & + & + & - & - & - & ++ & ++ \\
\hline
\end{tabular}


Les stérols et polyterpènes ainsi que les alcaloïdes sont présents dans chacun des extraits de plantes. Hormis l'extrait de Cyclosorus dentatus tous les autres contiennent des polyphénols et des flavonoïdes.

Selon N'Guessan et al. (2009), l'effet anti-asthmatique serait le fait des stérols et des alcaloïdes. Ces auteurs ont également signalé que les alcaloïdes tropaniques freineraient les sécrétions alvéolaires et calmeraient les crises d'asthme.

Bouquet et Debray (1974) ont montré que Microsorium punctatun est utilisée pour traiter la frigidité féminine liée à un sort ou naturelle ainsi que pour traiter certains œè̀mes. L'effet thérapeutique de cette plante contre les œdèmes pourrait être dû aux alcaloïdes. Selon N'Guessan et al. (2009), l'alcaloïde dénommé punamavine de goût amer est réputé pour traiter les maladies oculaires et est également indiqué contre les œdèmes. Bruneton (1999), rapporte cela en disant que les alcaloïdes indoliques exercent un effet antipaludique. L'effet antipaludique des substances végétales serait le fait des alcaloïdes. Cependant les enquêtes dans cette étude n'ont pas révélé cet effet antipaludique. Dans les travaux d'Adou (2007), les alcaloïdes n'ont pas été mis en évidence. Par contre, ils signalent la présence de quinones chez cette espèce, ce qui n'est pas le cas dans cette étude.

Selon N'Guessan et al. (2009), l'action anti-hypertensive signalée au cours de l'enquête serait le fait des polyphénols. Cet effet contre l'hypertension serait le fait des polyphénols probablement de type coumarines.

L'effet anti-rhumatismal des plantes serait dû aux flavonoïdes qui ont des propriétés anti-inflammatoires. Les propriétés anti-inflammatoires des flavonoïdes sont également signalées par Benguerba (2008) in Bouziane (2014). L'effet thérapeutique sur les enflures pourrait être le fait des flavonoïdes qui ont des propriétés anti-inflammatoires.

L'effet hémostatique dans le traitement des plaies est confirmé par Bouquet et Debray (1974) ; Adou et al., (2014). Cet effet serait le fait des tanins catéchiques qui ont des propriétés bactéricides.

Selon Kerharo et Bouquet (1950), toute la partie aérienne de Selaginella sp, pillée puis comprimée pour recueillir le jus est donné en lavement dans le traitement de la syphilis, tandis que la pulpe résiduelle est appliquée sur les chancres.

La présence de ces molécules dans ces espèces justifierait l'utilisation de ces plantes dans la pharmacopée traditionnelle. Selon plusieurs auteurs dont Zirihi (2006), ces molécules sont à la base de plusieurs propriétés pharmacologiques. Selon Nacoulma (1996), les quinones ont des propriétés antibactérien, antispasmodique, antiseptique, anthelmintique, cicatrisant, fongicide (mycose, dermatose). Ceci nous amène à penser que Lygodium smithianum pourrait avoir toutes ces propriétés du fait qu'elle renferme des quinones. 


\section{Conclusion}

Les enquêtes ethnobotaniques menées dans la forêt classée yabo-abbé sur les Pteridophytes dans la région d'Azaguié montrent que six (6) espèces de Ptéridophytes sont utilisées comme plantes médicinales sous différentes formes de préparations médicamenteuses contre divers maux. Les effets thérapeutiques sont produits par différents composés chimiques qui sont les stérols, polyterpènes, polyphénols, flavonoïdes, tanins catéchiques et les alcaloïdes. La présence de ces composés dans ces espèces justifierait l'utilisation de ces plantes dans la pharmacopée traditionnelle. En effet, selon plusieurs auteurs, ces molécules sont à la base de plusieurs propriétés pharmacologiques. Bien qu'il serait envisageable d'approfondir les études phytochimiques sur l'ensemble des Ptéridophytes et faire des évaluations sur des souches bactériennes et fongiques, nous pensons que l'objectif de départ de cette étude qui était de montrer les usages de ces taxons puis de faire un tri phytochimique (permettant d'appréhender les substances chimiques pouvant justifier les différentes activités) de celles signalées comme plantes médicinales est atteint.

\section{References:}

1. Adou LMD, 2000. Contribution à la connaissance de la flore de la Côte d'Ivoire: Étude taxonomique et écologique des Ptéridophytes du massif forestier Yapo-Abbé (Côte d'Ivoire). Mémoire de DEA l'Université FHB, UFR Biosciences, 101 p.

2. Adou LMD, 2007. Les Ptéridophytes du Sud-Est de la Côte d'Ivoire : systématique, écologie, biologie et ethnobotanique. Thèse de doctorat, Université Félix Houphouët-Boigny, Abidjan, Côte d'Ivoire. 254 p.

3. Adou LMD, Touré A, Komoé K, Ipou Ipou J, 2014. Nephrolepis biserrata, une Ptéridophyte utilisée comme plante médicinale en Côte d'Ivoire. Journal of Applied Biosciences, 81:7298-7306.

4. Adou LMD, Koné MW, Ipou Ipou J et N'Guessan KE, 2016. Ethnobotanique et analyse phytochimique qualitative de Pteridium aquilinum (L.) Kühn (Dennstaedtiaceae), une Ptéridophyte utilisée comme plante médicinale en Côte d'Ivoire. International Journal of Biological and Chemistry Sciences, 10(4): 1783-1792.

5. Aké-Assi L, 1984. Flore de la Côte d'Ivoire : étude descriptive et biogéographique, avec quelques notes ethnobotaniques. Thèse Doctorat, Université Nationale d'Abidjan, FAST, (Côte d'Ivoire), 1206 p.

6. Benguerba A, 2008. Étude Phytochimique et de la Phase Butanolique de L'espèce Inula crithmoides L. Thèse de Magister en Chimie Organique Option Phytochimie, Université Mentouri Constantine, Algérie, 91 p. 
7. Bodoua KO, 2013. Perceptions sociales et attitudes thérapeutiques associées aux césariennes indiquées en milieu rural de côte d'Ivoire. L'exemple des mères césarisées de la commune de la communauté Abbey d'Azaguié-Ahoua. DEA. Université Alassane Ouattara de Bouaké, 80 p.

8. Bouquet A \& Debray M, 1974. Plantes médicinales de Côte-d'Ivoire, Imprimerie Louis Jean, Paris (France), 232 p.

9. Bouziane C, 2014. Étude de l'interaction de la phospholipase A2 et flavanones isolées de l'Inule Visqueuse par modélisation moléculaire : Mémoire de Master, Université Abou Bekr Belkaid Tlemcen, Algérie, $46 \mathrm{p}$.

10. Bruneton J, 1999. Pharmacognosie, Phytochimie et plantes médicinales. Edition Technique et Documentation, $1120 \mathrm{p}$.

11. Doga D, 2017. Valorisation biotechnologique des légumineuses utilisées en médecine traditionnelle en côte d'Ivoire pour une agriculture durable. Thèse de Doctorat en sciences Agronomiques et Génie Rural. Institut National Polytechnique Félix Houphouët-Boigny (INP-HB), $152 \mathrm{p}$.

12. Enda Tiers-Monde, 1986. Encyclopédie médicinale de l'Afrique. Librairie de France. Volume IV, $1151 \mathrm{p}$.

13. Gonezieti BBHJ, 2016. Étude ethnobotanique des Ptéridophytes du Parc National du Banco. Mémoire de Master. Université Felix Houphouët-Boigny. Laboratoire de Botanique, $50 \mathrm{p}$.

14. Kakudidi E, 2004. Cultural and social uses of plants from and around Kibale National Park, Western Uganda. African Journal of Ecology, 42(1) : 114-118.

15. Kerharo J \& Bouquet A, 1950. Plantes médicinales et toxiques de la Côte-d'Ivoire Haute Volta. Vigot et Frères, Editeurs. Paris (VIè), 296 p.

16. Lukavsky K, 1996. Les fougères. Rapport des associés de la biodiversité. $\mathrm{n}^{\circ} 3$ Canada, $12 \mathrm{p}$.

17. Malay B, 2011. Ethno medicinal importance of some common Pteridophytes used by tribals of Ranchi and Latehar district of Jharkhand, India. The international quarterly journal of ethno and social sciences, 3(1/2) : 5-8.

18. Mangambu MJD, Diggelen RV, Mwanga JC, Ntahoba Yuka H, Malaisse F \& Robbrech TE, 2012 Étude ethnopteridologique, évaluation des risques d'extinction et stratégies de conservation aux alentours du Parc National de Kaluzi Biega (RD Congo), Géo-EcoTrop., $36: 137-158$.

19. Maregesi S.M., Ngassapa O.D., Pieters L. \& Vlietinck A., 2007. Ethnopharmacological survey of the Bunda district, Tanzania: Plants 
used to treat infectious diseases. Journal of Ethnopharmacology, 113 : 457-470.

20. Nacoulma OO, 1996. Plantes médicinales et pratiques médicales traditionnelles au Burkina Faso: cas du Plateau central, Thèse de Doctorat ès Sciences Naturelles, Université de Ouagadougou, Burkina-Faso, $605 \mathrm{p}$.

21. N'Guessan K, 1995. Contribution à l'étude ethnobotanique en pays Krobou (Côte d'Ivoire). Thèse de Doctorat de $3^{\mathrm{e}}$ cycle, Université Nationale de Côte d'Ivoire, FAST d'Abidjan, 557 p.

22. N'Guessan K, Tra Bi FH \& Koné MW, 2009. Étude ethnopharmacologique des plantes antipaludiques utilisées en médecine traditionnelle chez les Abbey et Krobou d'Agboville (Côte d'Ivoire), Ethnopharmacologia, 44 : 42-50.

23. Offoumou MR, 2015. Étude ethnobotanique des plantes médicinales utilisées pour le traitement du paludisme dans le département d'Agboville (Sud-Est de la Côte d'Ivoire). Mémoire Master, UFR Biosciences, Université de FHB, Côte d'Ivoire, 50 p.

24. Piba SC, Tra Bi FH, Konan D, Bitignon BGA \& Bakayoko A, 2015. Inventaire et disponibilité des plantes médicinales dans la forêt YapoAbbé, en Côte d'Ivoire. European Scientific Journal, 11(24): 161-181.

25. Rout SD, Panda T \& Mishra N, 2009. Ethnomedicinal studies on some pteridophytes of Similipal Biosphere Reserve, Orissa. India International Journal of Medicine and Medical Sciences, 23(5) : 192197.

26. Salhi S, Fadli M, Zidane L \& Douira A, 2010. Études floristique et ethnobotanique des plantes médicinales de la ville de Kénitra (Maroc). Lazaroa, 31: 133-146.

27. Singh S, Dixit RD \& Sahu TR, 2003. Some medicinaly important Pteridophytes of Central India. International Journal of Forestry Usuf. Management, 4 (2) : 41-51.

28. Tra-Bi FH, 1993. Les Ptéridophytes de la région d'Abidjan : Systématique, Anatomie et Écologie, Mémoire de DEA, Université de Cocody, Abidjan, 102 p.

29. Zirihi G. N., 2006. Études botanique, pharmacologique et phytochimique de quelques plantes Médicinales anti-paludiques et/ou immunogènes utilisées chez les Bété du Département d'Issia, dans l'ouest de la Côte d'Ivoire. Thèse de Doctorat d'État, UFR Biosciences, Université de Cocody Abidjan, Côte d'Ivoire, 126 p.

30. Zirihi GN, Kra AKM et Guédé-Guina F, 2003. Évaluation de l'activité antifongique de Microglossa pyrifolia Lam O. Ktze (Asteraceae) « PYMI » sur la croissance in vitro de Candida albicans. Revue Médicale et Pharmacopée Africaine, 17 : 1-19. 